\title{
Chirurgische Versorgung bei Tetraplegie
}

$V_{\text {er }}$ etzungsbedingte Läsionen des Rückenmarks im Halsbereich haben gravierende Funktionsverluste der unteren und oberen Extremitäten, einen Ausfall der sensorischen Innervation des gesamten Körpers und nachhaltige Störungen der systemischen Regulation zur Folge. Nur eine Minderheit der Patienten weist Partialläsionen mit potentieller Besserungstendenz auf. Die Mehrheit der Tetraplegiker muß sich auf Dauer mit der in allen Lebensbereichen wirksam werdenden Schwerstbehinderung arrangieren.

Dank der von Gutmann eingeführten Systematisierung der Behandlung in der Akut- und Postakutphase kann das Überleben der Schwerstverletzung heute regelmäßig sichergestellt werden. Die in spezialisierten Zentren durchgeführte funktionelle und psychosoziale Rehabilitation ermöglicht der Mehrzahl der Patienten eine Rückkehr in das familiäre Umfeld. Das sich ändernde Tätigkeitsspektrum der modernen Informationsgesellschaft kann auch Menschen mit schwersten funktionellen Handicaps eine sinnvolle berufliche Perspektive bieten.
Eine einmal gesetzte, pathoanatomisch definierte Rückenmarksläsion läßt sich in der Akutphase nur mit Einschränkungen und im weiteren Verlauf praktisch nicht mehr direkt chirurgisch beeinflussen. Eine Heilung im Sinne einer neuronalen Überbrückung des zerstörten Areals ist auf dem Stand der heute etablierten Therapie nicht induzierbar. Aktuelle, tierexperimentell validierte Therapieansätze mit einer Kombination aus neurogenen Wachstumsfaktoren und gleichzeitiger Blockierung der myelinen Inhibition durch Antikörper lassen für die Zukunft auf eine Behandlungsperspektive mit zumindest partieller Restitution der peripheren Innervierung hoffen.

Die chirurgische Therapie der Akutphase besteht in der Sicherung der Vitalfunktionen und der Stabilisierung der verletzten Halswirbelsäule unter Berücksichtigung der besonderen Gegebenheiten des Tetraplegikers. Dekompression des Spinalkanals und anschließende formwiederherstellende und stabile Spondylodese schützen das verletzte Halsmark vor weiteren Schäden, nutzen in Einzelfällen die verbliebenen Erholungschancen und stellen die immer notwendige Intensivtherapie mit uneingeschränkter Lagerungsmöglichkeit sicher. Oberste Priorität in der akuten Behandlungsphase hat die Vermeidung jedweder additiver Schädigungen bei Bergung, Transport und operativer Versorgung.
Die postakute Phase mit der aufwendigen Überwachungs- und Lagerungstherapie sowie der frühzeitigen Einleitung der Rehabilitation sollte immer in den nunmehr flächendeckend und kapazitär ausreichend vorhandenen Zentren für die Behandlung Rükkenmarkverletzter durchgeführt werden. Demgegenüber wird die Erstaufnahme aus den logistischen Notwendigkeiten der Notfallversorgung heraus häufig in Traumazentren erfolgen. Diese sollten über eine umfassende Kompetenz und durchgehende Dienstbereitschaft für die Versorgung von Wirbelsäulenverletzungen verfügen und darüberhinaus die speziellen Aspekte für die Behandlung Gelähmter kennen und respektieren.

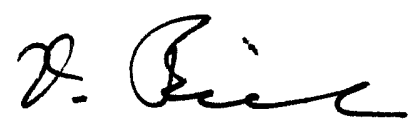

V.Bühren 